\title{
Acid Activated Palygorskite: An Efficient Solid acid Catalyst for Acetylation Reactions - A Comparative Study
}

\author{
DHANYA BALAN A ${ }^{1}$ and PUSHPALETHA ${ }^{1 *}$ \\ Department of Chemistry, Government College Kasaragod, Kerala-671123, India. \\ *Corresponding author E-mail: ppletha @ rediffmail.com
}

http://dx.doi.org/10.13005/ojc/340655

Received: July 06, 2018; Accepted: November 25, 2018)

\begin{abstract}
Mild and efficient solid acid catalysts were prepared from natural palygorskite collected from Karnataka and Hyderabad and compared their catalytic activities using the acetylation reaction. Modification technique used for the preparation of the catalysts are acid-activation. During acid-activation exchangeable cations are replaced by $\mathrm{H}^{+}$ions and a part of octahedral cations are dissolving and thus creating new acid sites in the crystal. The catalytic performances of these catalysts were investigated by using the acetylation reaction. Acetylation reaction was done by using different primary and secondary alcohol. Physicochemical properties were characterized by XRD, SEM, $\mathrm{NH}_{3}$-TPD measurements.
\end{abstract}

Keywords: Acetylation, Palygorskite, Acid-activation, Cation exchange capacity.

\section{INTRODUCTION}

Palygorskite is a Hydrated Magnesium Alumino Silicate Hydroxide mineral with a fibrous morphology. This clay has been mined for centuries because of its many useful properties such as large surface area and micro porosity. ${ }^{1,2}$ It has many commercial uses in the fertilizer, Pharmaceutical and pesticide industries. The search of new application of palygorskite as either catalyst or catalyst support material is still ongoing. Palygorskite with the appropriate treatment could be converted in to an efficient solid acid catalyst for acetylation reaction. Acetylation is an important industrial organic reaction and is applied on a large scale in the chemical industry.
Other reports can be found on the implementation of such solid acids for acetylation reactions. Zeolites, ${ }^{3-5}$ supported heteropoly acids on silica and silica-zirconia ${ }^{6}$, mesoporous supported Lewis and Bronsted acids ${ }^{7-9}$, aluminium phosphate or molecular sieves ${ }^{10}$, sulphated zirconia ${ }^{11}$, ion exchange resins ${ }^{12}$, and Lewis acids ${ }^{13}$ have been reported to be active in acetylation reaction.

The catalyst prepared by the modification of palygorskite was reported to catalyze reactions like polymerization of caprolactone ${ }^{14}$, oxidation of ethanol ${ }^{15}$ and styrene ${ }^{16}$, synthesis of alcohol ${ }^{17}$ etc. Acid activated attapulgate was found to be an efficient solid acid catalyst for acetylation reaction ${ }^{18}$. India has large deposits of palygorskite in different

This is an Open Access article licensed under a Creative Commons license: Attribution 4.0 International (CC- BY). Published by Oriental Scientific Publishing Company @ 2018

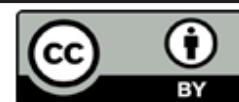


states. Only few reports are found on the catalytic activity of these clays. Here we compare the catalytic potential of solid acid catalysts developed from natural palygorskite collected from Karnataka and Hyderabad using the modification method of acid-activation and thus comparing their catalytic activities.

\section{MATERIALS AND METHODS}

\section{Clay Samples and Chemicals Used in the Present Investigation}

Clay mineral used for the present study was procured from Korvi Fuller's Earth limited Karnataka and Ashapura mine chemicals Gujarath. Chemicals used were sulphuric acid, ferric chloride, stannous chloride, manganese chloride, sodium hydroxide, hydrochloric acid, sulphuric acid, n-hexane, n- heptane, 2-butane, lauryl alcohol etc.

\section{Purification of clay sample}

Purification was done by suspending the clay in water, stirring vigorously and allowing the coarser particles to settle. The fines have been passed through a $360 \#$ B.S sieve to separate the clay particles of size less than 45 micrometers. The slurry is dried and the dry mass is disintegrated.

\section{Preparation of the catalyst}

The method used for the preparation of the catalyst is acid-activation of the clay mineral.

\section{Acid-activation of the clay}

Procedure: Acid-activation was done by refluxing the purified sample with the sulphuric acid solution of different concentration in 1:4 ratios in a 250 $\mathrm{ml}$ R.B flask with the condenser for $\mathbf{4 5}$ minutes. It was cooled and added to $1 \mathrm{~L}$ water and allowed to settle and decanted. It was then filtered and washed free of anions. The sample was dried in an air oven at $110^{\circ} \mathrm{C}$ for 6 hours. Catalysts prepared are listed in Table 1.

\section{Techniques used for the characterization of catalysts}

$X$ ray diffraction $(X R D)$ was performed on D8 advance X-ray diffractometer. Morphological studies were carried out by scanning electron microscopy. The catalysts were characterized by acidity measurements. Clays contain both Bronsted and Lewis acid sites. Sodium hydroxide titration and $\mathrm{NH}_{3}$-TPD another characterization technique used to study the acidity of the clay minerals.

\section{Catalytic activity measurements}

Activity of the catalyst was monitored by acetylation reaction. The reaction was carried out in an R.B flask fitted with condenser. In acetylation, reaction mixture consisting of cyclohexanol (0.05 mol) and acetic acid (excess) was refluxed in the presence of $0.5 \mathrm{~g}$ catalyst. After the reaction was stopped, the catalyst was separated by filtration and analyzed by Gas Chromatograph.

\section{RESULTS AND DISCUSSION}

\section{Characterization of the Catalyst \\ Powder X-ray diffraction studies}

$X R D$ is used for the identification of the clay mineral present in the sample. It gives information about the changes in crystallinity during acid-activation. It also gives information about the presence of impurities, if any, in the sample. Powder X-ray diffraction patterns of the raw and acid activated sample (Fig.1) indicate that the major mineral in the sample is palygorskite. The peaks at $2 \theta$ value of 8.6 are characteristics of palygorskite mineral. There is a peak at $2 \theta$ value of 20 and 26.9 which indicates the presence of impurity quartz. The sample was found to be reasonably pure but for traces of carbonate as impurities ( $2 \theta$ value 29.49). Carbonate impurity dissolves on acid-activation and thus the peak disappeared.

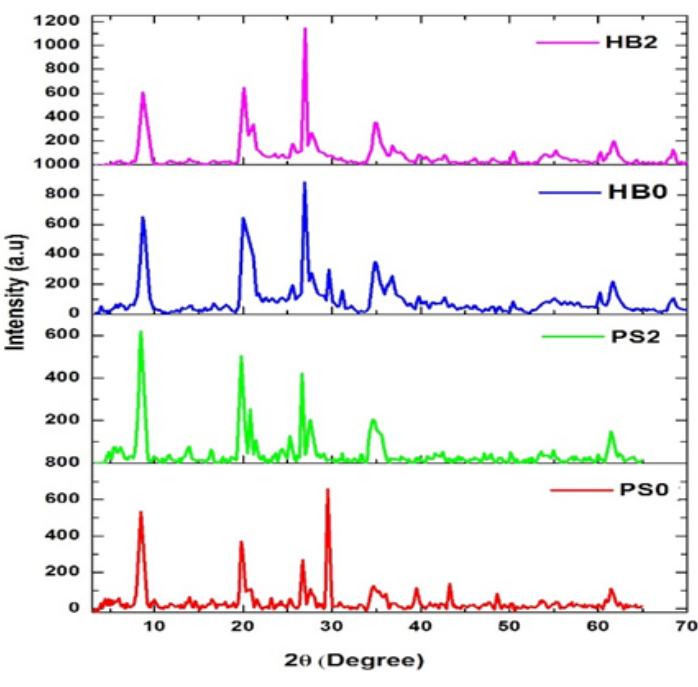

Fig. 1. XRD pattern of raw and acid activated palygorskite sample Scanning electron microscopy Analysis

Morphological studies were carried out by scanning electron microscopy (Fig. 2). SEM micrographs of the raw sample show elongate lath 
shaped crystals and their bundles, characteristics of palygorskite mineral. SEM photographs of the acid treated samples show that the acid treatment has not affected the fibrous morphology of palygorskite. Even after the removal of octahedral layer fibrous morphology is maintained indicating the retention of order in the tetrahedral layer in palygorskite.

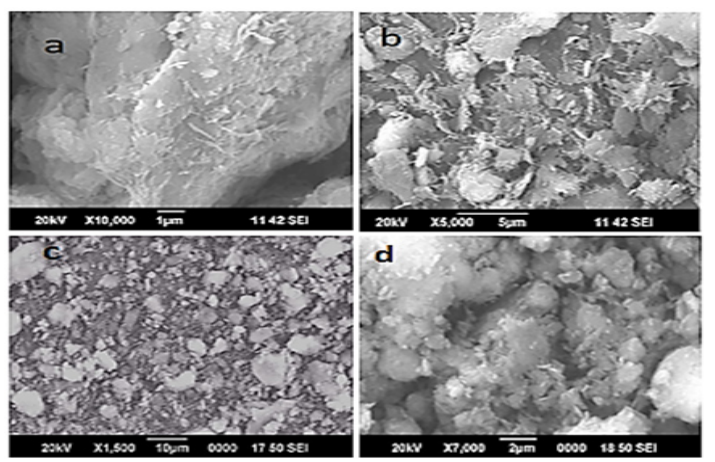

Fig. 2. S E M of raw and acid activated palygorskite. (a) SEM of raw sample collected from Karnataka(b) SEM of raw sample collected from Hyderabad. (c) S E M of acid activated samples collected from Hyderabad. (d) SEM of acid activated sample collected from Karnataka. Both acid activated samples having more porous structure than the rawsample but fibrous morphology is maintained

\section{Acidity measurements}

Acidity of clay surface can be defined as the ability to convert an adsorbed neutral base to its conjugate acid; it is the most important parameter, which controls the catalytic activity of clay minerals. Clays contain both Bronsted and Lewis acid sites. Different methods are used to measure the acidity of clay minerals.

$\mathrm{NH}_{3}$-TPD is an important technique used for the characterization of catalysts and gives information about the acidity of catalysts. It gives concentration of both Lewis and Bronsted acid sites. Acidity is the most important parameter that decides the activity of the catalyst. The $\mathrm{NH}_{3}$-TPD was carried out to evaluate the acid site distribution of the raw and acid activated palygorskite mineral (Fig. 3). TPD profiles of raw and acid activated palygrskite are based on the desorption temperature maxima which could be divided in to three regions, corresponding to (a) Intermediate or medium (100$\left.200^{\circ} \mathrm{C}\right)$, (b) strong $\left(200-400^{\circ} \mathrm{C}\right)$, and very strong acid strengths $\left(>400^{\circ} \mathrm{C}\right)$. Acid activated palygrskite exhibits three peaks at $100^{\circ} \mathrm{C}, 450^{\circ} \mathrm{C}$ and $660^{\circ} \mathrm{C}$. The three resulting peaks can be correlated to three different types of active sites. The first and second peak suggests that palygorskite possesses a large number of acid sites with intermediate and strong acid strength. The peak at $650^{\circ} \mathrm{C}$ corresponds to very strong acid strengths. The total acid sites of HB2 are greater than those of raw and other acid activated samples. Thus HB2 shows more activity.

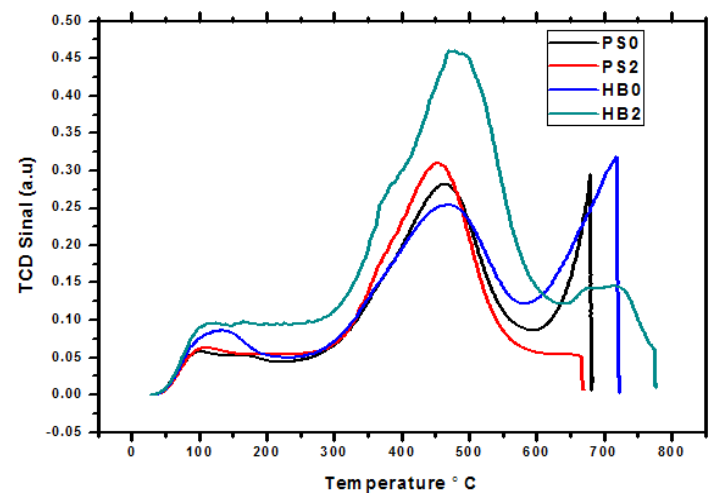

Fig. 3. $\mathrm{NH}_{3}$-TPD of raw and acid activated sample Table 1: Acid-activation using different concentration of sulphuric acid

SI Concentration catalyst support Catalyst support No of sulphuric acid(Sample collected (Sample collected solution (N) from Karnataka) from Hyderabad)

\begin{tabular}{lcll}
\hline 1 & 0 & PS0 & HB0 \\
2 & 0.1 & PS1 & HB1 \\
3 & 0.5 & PS2 & HB2 \\
4 & 1.0 & PS3 & HB3 \\
5 & 1.5 & PS4 & HB4 \\
6 & 2.00 & PS5 & HB5 \\
\hline
\end{tabular}

The acidity was measured by titration with sodium hydroxide solution. Surface acidity calculated by sodium hydroxide titrations is given in Table 2. Acid properties of clay minerals stem from the terminal hydroxyl groups, bridging oxygens and from the dissociation of interlayer water molecules coordinated to the exchangeable cations. There is a remarkable increase in acidity on acid-activation which is shown in Table 2. Acidity of raw sample collected from Korvi fullers earth limited Karnataka is only 162.64 milliequiv./g and the raw sample collected from Hyderabad is 200.35 milliequi/g. On acid-activation using $0.1 \mathrm{~N} \mathrm{H}_{2} \mathrm{SO}_{4}$ acidity increases to 193.63 milliequiv./g for Karnataka sample and acidity increases to 332.55milliequiv./g for Hyderabad sample. Acidity increases with acid concentration used for activation. It reaches a maximum value and then shows a decrease. The maximum value is shown by PS2 and HB2, clay activated with 
$0.5 \mathrm{NH}_{2} \mathrm{SO}_{4} 332.55$ milliequiv./g. From there acidity decreases with increase in concentration of sulfuric

acid solution. Thus acidity first increases to a maximum value and then decreases.

Table 2: Surface acidity, Cation exchange capacity and percentage of conversion of raw and acid activated samples

\begin{tabular}{lcccccccc}
\hline $\begin{array}{l}\text { SI. } \\
\text { No. }\end{array}$ & $\begin{array}{c}\text { Catalyst Surface acidity } \\
\text { (milliequiv./g) }\end{array}$ & $\begin{array}{c}\text { CEC } \\
\text { (milliequiv./100g) }\end{array}$ & \multicolumn{2}{c}{$\begin{array}{c}\text { Conversion (\%) Catalyst } \\
\text { Acetylation }\end{array}$} & $\begin{array}{c}\text { Surface acidity } \\
\text { (milliequiv./g) }\end{array}$ & $\begin{array}{c}\text { CEC } \\
\text { (milliequiv./100g) }\end{array}$ & $\begin{array}{c}\text { Conversion (\%) } \\
\text { Acetylation }\end{array}$ \\
\hline 1 & PS0 & 162.64 & 48.34 & 60.43 & HB0 & 200.35 & 54.81 & 66.86 \\
2 & PS1 & 193.63 & 60.73 & 68.42 & HB1 & 289.00 & 63.50 & 74.87. \\
3 & PS2 & 200.30 & 72.89 & 76.34 & HB2 & 332.55 & 78.23 & 84.90 \\
4 & PS3 & 191.74 & 54.87 & 69.77 & HB3 & 296.05 & 63.04 & $73 . .02$ \\
5 & PS4 & 169.49 & 50.53 & 67.09 & HB4 & 274.36 & 52.66 & 71.54. \\
6 & PS5 & 184.90 & 42.22 & 66.85 & HB5 & 267.99 & 48.00 & 65.01 \\
\hline
\end{tabular}

\section{Cation Exchange Capacity measurements}

The clay minerals have a property of adsorbing certain anions and cations and retaining them in an exchangeable state. The heat evolved in the course of an ion exchange reaction is usually small, $\sim 2 \mathrm{kcal} /$ mole. In clay minerals the commonest exchangeable cations are $\mathrm{Ca}^{2+}, \mathrm{Mg}^{2+}, \mathrm{K}^{+}, \mathrm{NH}^{4+}$ and $\mathrm{Na}^{+}$.

The property of ion exchange and the exchange reactions are of very fundamental and practical importance in all the fields in which clay minerals are studied and used because the physical properties of clay materials are dependent on a large extent on the exchangeable ions associated with the clay. Cation exchange capacity $(C E \mathrm{C})$ is found to control the catalytic activity just like acidity. $\mathrm{C} \mathrm{E} \mathrm{C} \mathrm{of} \mathrm{clays} \mathrm{was} \mathrm{determined} \mathrm{by} \mathrm{exchanging} \mathrm{the}$ cations of the clay by ammonium ions and measuring the amount of ammonium ions exchanged. Cation exchange capacity of raw and acid activated sample is listed in Table 2. Cation exchange capacity of PSO is 48.34 milliequiv. $/ 100 \mathrm{~g}$ and for HBO is 54.81 milliequiv./100g. During acid-activation cation exchange capacity first increases and reaches a maximum and then decreases. Maximum cation exchange capacity was shown by the sample treated with $0.5 \mathrm{~N} \mathrm{H}_{2} \mathrm{SO}_{4}$ (72.89 milliequiv./100g and 78.23 milliequiv. $/ 100 \mathrm{~g}$ ). With the severity of acid treatment crystal structure starts collapsing and thus cation exchange capacity decrease.

\section{Catalytic activity measurements}

The catalytic activity of raw and acid activated catalyst was studied by acetylation of cyclohexanol using acetic acid.Acetylation was done batch wise in liquid phase by refluxing 0.05 mole of cyclohexanol with 0.05 mole of acetic acid in presence of $0.5 \mathrm{~g}$ of the acid activated catalyst. The percentage of conversion were given in Table 3.
Table 3: Catalytic activity measurements

\begin{tabular}{lccc}
\hline Substrate & \multicolumn{3}{c}{ Conversion (\%) } \\
\hline & PS2 & HB2 & AM2 \\
\hline Cyclohexanol & 76.34 & 84.90 & 77.90 \\
Benzyl alcohol & 74.30 & 82.69 & 76.00 \\
1-Butanol & 64.58 & 72.11 & 66.10 \\
1-Hexanol & 88.00 & 98.36 & 89.93 \\
1-Heptanol & 86.00 & 94.32 & 86.98 \\
1-Octanol & 85.39 & 90.61 & 87.64 \\
Lauryl alcohol & 51.83 & 78.21 & 52.43 \\
Butane-2-ol & 44.38 & 73.73 & 46.23 \\
\hline
\end{tabular}

Among various catalyst prepared by acid-activation of palygorskite, maximum acidity is obtained in the case of $0.5 \mathrm{~N} \mathrm{H}_{2} \mathrm{SO}_{4}$ treated sample (PS2 and HB2). Comparing the percentage of conversion of samples collected from two different places maximum conversion is obtained in the case of Hyderabad sample.

The effect of reaction time on catalytic activity of acid activated palygorskite was monitored by changing the reaction time. Reaction was carried out in a R. B. flask fitted with condenser. In each experiment, $0.5 \mathrm{~g}$ of the catalyst was added to the reaction mixture. Acetylation was carried out by using cyclohexanol and acetic acid. The percentage of conversion was measured after various times using gas chromatography and is given in Table 3 . Maximum conversion was obtained in 3 hours.

Effect of catalyst loading on the percentage conversion was also monitored by using the reaction acetylation of cyclohexanol using acetic acid. Results are given in Table 4. The minimum amount of acid activated catalyst required for the conversion is 0.5 $\mathrm{g}$ for 0.05 mole alcohol. 
Table 4: Effect of reaction time, Effect of catalyst loading and Reusability of the catalyst

\begin{tabular}{|c|c|c|c|c|c|c|c|c|c|c|c|}
\hline \multicolumn{3}{|c|}{ Effect of Reaction Time } & \multicolumn{4}{|c|}{ Effect of catalyst loading } & \multicolumn{5}{|c|}{ Reusability of the catalyst } \\
\hline $\begin{array}{l}\text { Catalyst } \\
\text { loading } \\
\text { (g) }\end{array}$ & $\begin{array}{l}\text { Reaction } \\
\text { time } \\
\text { (h) }\end{array}$ & $\begin{array}{c}\text { Conversion } \\
\text { (\%)PS2 }\end{array}$ & $\begin{array}{c}\text { Conversion } \\
\text { (\%)HB2 }\end{array}$ & $\begin{array}{l}\text { Catalyst } \\
\text { loading } \\
\text { (g) }\end{array}$ & $\begin{array}{l}\text { Reaction } \\
\text { time } \\
\text { (h) }\end{array}$ & $\begin{array}{c}\text { Conversion } \\
\text { (\%)PS2 }\end{array}$ & $\begin{array}{c}\text { Conversion } \\
\text { (\%)HB2 }\end{array}$ & $\begin{array}{l}\text { Catalyst } \\
\text { loading } \\
\text { (g) }\end{array}$ & $\begin{array}{c}\text { Reaction } \\
\text { time } \\
\text { (h) }\end{array}$ & $\begin{array}{c}\text { Conversion } \\
\text { (\%)PS2 }\end{array}$ & $\begin{array}{c}\text { Conversion } \\
(\%) \mathrm{HB} 2\end{array}$ \\
\hline 0.5 & 1 & 65.86 & 75.22 & 0.25 & 3 & 65.75 & 78.64 & 0.5 & 3 & 76.34 & 84.9 \\
\hline 0.5 & 2 & 70.43 & 80.61 & 0.5 & 3 & 76.34 & 84.90 & 0.5 & 3 & 73.57 & 83.00 \\
\hline 0.5 & 3 & 76.34 & 84.90 & 0.75 & 3 & 75.81 & 84.00 & 0.5 & 3 & 68.69 & 79.98 \\
\hline 0.5 & 4 & 76.12 & 84.00 & 1.00 & 3 & 70.41 & 80.04 & 0.5 & 3 & 66.42 & 78.65 \\
\hline
\end{tabular}

Reusability of the catalyst was also tested by using the same reaction. No reduction in percentage of conversion was noticed even after four cycles. The results were given in Table 3 .

Comparison of activity of the catalysts with catalysts prepared from standard sample collected from clay mineral repository

Acidity, Cation exchange capacity and the activity of the catalyst is compared with catalyst prepared from the original sample (AM) collected from Clay mineral society of Attapulgate, Department of Geology PFL1 US Repository, Missori Columbia. Among the acid activated samples, PS1 to PS5, PS2 (0.5 N Sulphuric acid treated sample) showed maximum activity. So the standard sample (AM2) was also treated with $0.5 \mathrm{~N}$ sulphuric acid. The acidity and cation exchange capacity values of AM2 was found to be comparable with the acidity and cation exchange capacity values of the sample collected from Karnataka (PS2) and Hyderabad (HB2). The catalytic activity values of these samples are also comparable with the sample collected from Karnataka and Hyderabad.

\section{CONCLUSION}

Among various catalysts prepared by acid-activation of palygorskite maximum acidity is obtained in the case of $0.5 \mathrm{~N} \mathrm{H}_{2} \mathrm{SO}_{4}$ sample. Acid activated palygorskite was found to be an efficient catalyst for acetylation reactions. Comparing the catalytic activities of both Karnataka and Hyderabad sample maximum conversion was obtained in the case of Hyderabad sample.The optimum time and catalyst loading for maximum yield was found to be $3 \mathrm{~h}$ and $0.5 \mathrm{~g}$ for 0.05 mole of the reactants. In conclusion, modified natural palygorskite collected from two different places were found to be eco-friendly, in expensive and reusable heterogeneous catalyst for acetylation of primary and secondary alcohols. By comparing the catalytic activity values of both Hyderabad and Karnataka sample clay mineral Collected from Hyderabad was found to be a more efficient catalyst for acetylation reaction.

\section{ACKNOWLEDGEMENT}

The authors gratefully acknowledge the financial support provided by state council for science technology and environment under "Back to lab program".

\section{REFERENCES}

1. Artioli, G.; Galli, E. Mater. Sci. Forum., 1994, 166, 647-652.

2. Giustetto,R.; Compagnon, R. Clay miner., 2011, 46, 371-385.

3. Freese, U.; Heinrich, F.; Roessner, F. Catal. Today., 1999, 49, 237-244.

4. Rohan, D.; Canaff, C., Fromentin, E.; Guisnet, M. J. J. Catal.,1998,177, 296-305.

5. Smith, K.; Zhenhua, Z.; Hodgson, P. K. G. J. Mol. Catal. A: Chem.,1998, 134, 121-128.

6. Bachiller-Baeza, B.; Anderson, J. A. J. Catal.,
2004, 228, 225-233.

7. Yadav, G.D.; Pujari, A. A. Green Chem., 1999, 1, 69-74.

8. Yadav, G.D.;Krishnan, M.S. Chem. Eng. Sci., 1999, 54, 4189-4197.

9. Yadav, G.D.; Asthana, N.S.Ind. Eng. Chem. Res., 2002, 41, 5565-5575.

10. Zhao, Z. J. Mol. Catal. A: Chem., 2000, 154, 131-135.

11. Yadav, G.D.; Metha, P. Ind. Eng. Chem.Res., 1994, 33, 2198-2208. 
12. Saha, B., Chopade, S.; Mahajani, S. Catal. Today., 2000, 60, 147-157.

13. Procopiu, P., Baugh S., Flask, S.; Ingliss, G. J. J. Org. Chem.,1998, 63, 2342-2347.

14. Wang, G.; Ma, R.; Chen, T.; Yan, C.; Gao, J.; Bao, F. Polymer Plastic Technol. Eng., 2013, 52, 1193-1199.

15. Wang, K.; Wang, H.; Pasupathi, S.; Linkov, V.; ji, S.; Wang, R. Electro Chim. Acta., 2012, 70,
394-401.

16. Wang, F.; Zhang, J.; Liu, C.; Liu, J. Appl. Clay Sci., 2015, 105, 150-155.

17. Guo, H.; Zhang, H.; Peng, F.; Yang, H.; Xiong, L.;Huang, C.; Wang, C.; Chen, X.; Ma, L.; Appl. Clay Sci., 2015, 111, 83-89.

18. Pushpaletha, P.; Lalithambika, M. Appl. Clay Sci., 2010, 51, 424-430.

19. A, M.; Appl. Clay Sci., 2010, 51, 424-430. 